\title{
A Fast Algorithm for Finding the Non Dominated Set in Multi objective Optimization
}

\author{
K.K.Mishra and Sandeep Harit \\ MNNIT,Allahabad,India
}

\section{INTRODUCTION:}

The working of single objective optimization algorithm and multi objective optimization algorithm is quite different. This difference is due to number of optimal solution approached by both the algorithms. In single objective optimization problem there will be a single optimal solution, even though in multi model optimization there may be more than one solution but we are interested in only one optimal solution, where as in multi objective optimization problem, there will be many set of optimal solutions. These sets are called different non dominated front, and every non dominated front will contain a set of non dominated solutions thus there are two tasks of an ideal multi objective optimization algorithm (i) To find multiple non dominated fronts (or to identify different set of non dominated solutions). (ii) To seek for Pareto optimal solutions with a good diversity in objective and decision variable values.

In this paper, we explain a new algorithm for finding non dominated set of a multi objective optimization problem. In literature many algorithms are used for this task like naïve and slow method [2], fast and efficient method [3] and Kung et al method [7]. Recently two new algorithms are proposed by Ding [4] and Jun Du[1].The worst case time complexity of all algorithm(including recently proposed algorithm) is $\left(\mathrm{OM}(\mathrm{N})^{2}\right)$, Previously Kung's algorithm was best in its average and best case time complexity but Jun du in 2007 proved that his algorithm is best in comparison of kung's algorithm. While we were unable to reduce worst case time complexity, The best case time complexity of proposed algorithm is $\mathrm{O}(\mathrm{NLog}(\mathrm{N}))$ ( for any number of objective functions )which is a improvement as compared to othere algorithms. Also it follows a simple approach, no hectic summation and production method.

The paper is organized into four sections. Section 2 presents some background detail of Different Preexisting Algorithms and specifies the necessary definition related to non dominated set. Section 3 describes the proposed approach and stepwise algorithm also difference between Kung's algorithm and proposed algorithm is presented; In Section 4, an experimental analysis and complexity of the proposed algorithm are presented. Finally, Section 5 concludes the paper.

\section{BACKGROUND}

\subsection{Dominance and Pareto-Optimality}

Most multi-objective optimization algorithms use the concept of dominance in their search. Here, we define the concept of dominance and related terms and present a number of techniques for identifying dominated solutions in a finite population of solutions. Definition of dominated points and non-dominated set are given below

Definition 1: A solution $\mathrm{x}^{(1)}$ is said to dominate the other solution $\mathrm{x}^{(2)}$, if both conditions 1 and 2 are true:

1. The solution $\mathrm{x}^{(1)}$ is no worse than $\mathrm{x}^{(2)}$ in all objectives, or $\mathrm{f}_{\mathrm{j}}\left(\mathrm{x}^{(1)}\right) \mathrm{f}_{\mathrm{j}}\left(\mathrm{x}^{(2)}\right)$ for all $\mathrm{j}=1,2 \ldots \mathrm{M}$.

2. The solution $x^{(1)}$ is strictly better than $x^{(2)}$ in at least one objective, or $\mathrm{f}_{\mathrm{j}}\left(\mathrm{x}^{(1)}\right){ }^{4} \mathrm{f}_{\mathrm{j}}\left(\mathrm{x}^{(2)}\right)$ for at least one $j \in\{1,2 \ldots$... $\}$.

Definition 2: (Non-dominated set): Among a set of solutions $\mathrm{P}$, the non-dominated set of solutions $\mathrm{P}$ ' are those that are not dominated by any member of the set $P$

\subsection{Review of some standard algorithms}

Before discussing the proposed algorithm, let us review some preexisting algorithms.

\subsubsection{Kung's Algorithm:}

Kung algorithm is the most efficient and widely used one. In this approach we have to first sort the population in decending order in accordance to first objective function. Thereafter, the population is recursively halved as top(T) and bottom(B) subpopulations. As Top half (T) is better in objective in comparison to Bottom half (B) in first objective, so we check the bottom half for domination with top half .The solution of B which are not dominated by solutions of $\mathrm{T}$ are merged with members of $\mathrm{T}$ to form merged population M.The complete algorithm is given below:

Step 1: Sort the population according the descending order of importance in the first objective function and rename the population as $\mathrm{P}$ of size $\mathrm{N}$.

Step 2 :Front $(\mathrm{P})$ : if $|\mathrm{P}|=1$, return $\mathrm{P}$ as the output of Front $(\mathrm{P})$.Otherwise, $\mathrm{T}=\operatorname{Front}\left(\mathrm{P}^{(1)}-\mathrm{P}^{(\mid \mathrm{P} / 2)}\right)$ and $\mathrm{B}=$ Front $\left(\mathrm{P}^{(\mid \mathrm{P} / 2+1)}-\mathrm{P}^{(\mathrm{P})}\right)$. IF $\mathrm{i}^{\text {th }}$ non -dominated solution $\mathrm{B}$ is not dominated by any non-dominated solution of $\mathrm{T}$, create a merged set $\mathrm{M}=\mathrm{T} \mathrm{U}$ i. Return $\mathrm{M}$ as output of Front $(\mathrm{P})$.

2.2.2Sorting based algorithm (Jun Du Algorithm):

This algorithm is given in two steps as follows:

Step 1: the population of solutions is sorted according to the descending order to every objective functions. Thereafter, several solutions sequences could be presented and each of them corresponds to one objective. Every solution could be scored according to the position it takes, higher score it gains. Then, each solution could get various 
scores corresponding to various objectives. Take the scores summation of every solution, and sort it to get one new solution summation sequence. The new summation sequence could be used for finding non dominated set by deleting all the dominated solution inside.

Step 2: The bottom of the summation sequence is used as the start of the compared solution, while the top of the summation sequence is used as the start of the comparing one. Once the compared solution is observed by any one anterior to it, it is deleted. When the compared solution becomes the top one of the sequence, the dominated set is drawn out.

2.2.3Ding's algorithm: Based on the definitions of indices, rank set and propositions given in paper 4, the following procedure is used by Ding which identifies the non dominated solutions. Interested reader may refer paper[4] for further study.

Algorithm Identifying the Non-dominated Set: MLNFC.

Input: $X$.

Output:The non-dominated set $-X 0$ of $X$

( $R 0$ store the non-dominate set of rank set $R$ ).

Using quick-sort method to create indices I1; I2; :::; IM

and

rank set $R$;

Initiate R0 with I1(1)0s, I2(1)0s, .., IM(1)0s, eliminate

duplicate ones;

for $(i=1 ; i \cdot N ; i++)$ Set $r(\mathrm{i})$ not checked;

stop $=N ; / /$ Set initial termination position $\mathrm{N}$;

for $(i=2 ; i \cdot s t o p ; i++) \mathrm{f}$

//Search at the ith entries of the indices;

for $(j=1 ; j \cdot M ; j++) \mathrm{f} / /$ Search at the ith entry of index $\mathrm{Ij}$;

if(Ij(i) $0 \mathrm{~s}$ not checked $) \mathrm{f}$

Compare it with the non-dominated ones among

Ij(1)0s; Ij(2)0s; :::; Ij(i i 1)0s and set it checked;

if(Ij(i) $0 \mathrm{~s}$ is not dominated by any of them $) \mathrm{f}$

put it in $R 0$;

i0 = max of its ranks(or components);

stop $=$ minfstop, $\mathrm{i} 0 \mathrm{~g} ; / /$ Update termination position;

\}

\}

\}

\}

3. PROPOSED ALGORITHM: The proposed approach is very different from existing algorithms. In existing algorithms, we are able to classify solutions only after finding all dominated solutions. So to find non dominated solutions, we need to search entire set repeatedly. In proposed algorithm, we store only non dominated solutions. We perform a few comparisons to classify a point in to either dominated set or non dominated set. We first sort population according to the descending order of importance to the first objective value. In this way the solutions which are good in first objective will come first, in the list those having bad value will come in last. We initialize a set $\mathrm{S} 1$, for keeping non dominated solutions only. We start with the first solution and add this solution to non dominated set S1. Since first point is best in terms of first objective so no point can dominate this point in first objective, so it will be non dominated. Now we compare every other solution of the list with this set S1 and update this set when we find another non dominated solution and skip on those solutions which are dominated by any element of the set. For example if solutions in the list are unique in first objective function value, then for second point we need only one comparison to decide whether this is dominated or non dominated. The reason can be explained as follows, this solution can be dominated by only first solution (which is best in first objective).it can not be dominated by other solutions because its value for first objective function is greater than all solutions except first. Similarly for the third solution we need at most two comparisons from fist and second point. And for the last point of list we need to compare this solution to all non dominated solutions. If solutions in list are not unique in first objective function value, then we have to make certain modification in proposed algorithm. Like we have to check every solution to its immediate successors, if any immediate solution dominates this solution then we have to remove this point from the non dominated set S1.Finally we display the non dominated solutions.

\subsection{Step by step procedure for the proposed algorithm}

The proposed algorithm can be executed using the following steps.

1. Sort all the solutions $\left(\mathrm{P}_{1} \ldots \mathrm{P}_{\mathrm{N}}\right)$ in decreasing order of their first objective function (F1) and create a sorted list $(\mathrm{O})$

2. Initialize a set $S_{1}$ and add first element of list $\mathrm{O}$ to $\mathrm{S}_{1}$

3. For every solution $\mathrm{O}_{\mathrm{i}}$ (other than first solution ) of list $\mathrm{O}$, compare solution $\mathrm{Oi}$ from the solutions of $\mathrm{S}_{1}$

i. If any element of set $\mathrm{S}_{1}$ dominate $\mathrm{O}_{\mathrm{i}}$, Delete $\mathrm{O}_{\mathrm{i}}$ from the list

ii. If $\mathrm{O}_{\mathrm{i}}$ dominate any solution of the set $\mathrm{S}_{1}$, Delete that solution from $\mathrm{S}_{1}$

iii. If $\mathrm{O}_{\mathrm{i}}$ is non dominated to set $\mathrm{S}_{1}$, Then update set $\mathrm{S}_{1}=\mathrm{S}_{1} \mathrm{U} \mathrm{O}_{\mathrm{i}}$

iv. If set $\mathrm{S} 1$ becomes empty add immediate solution at immediate solution to $\mathrm{S} 1$

4. Print non dominated set $\mathrm{S}_{1}$

\subsection{How Proposed Algorithm is Different from Kung's Algorithm:}

In First look the working of proposed algorithm resembles with Classic Kung's algorithm but it is quite different from Kung's algorithm. This difference is due to deleting procedure of both the algorithm. In Kung's algorithm a recursive approach was used to delete the dominated points from the set, this approach gives no weight age to the knowledge that is earned by sorting the solutions according to their first objective. Let us make it clear, when we sort the solutions according to their first objective. The solutions which have a good probability to dominate other solutions will come on the beginning of the list. This can be understand by the definition of dominated point, If a point dominate other point it has to be good in all objectives so it 
will be defiantly good in first objective. That is what we are doing when we apply sorting to the list. So it would be better if we start deleting points from the front end of the sorted list. In this way, we will not waste our time in making unnecessary comparisons as was done in Kung's algorithm. In our algorithm we give proper weight age to the knowledge earned by sorting and our deleting procedure start with the front end of the list. Let as take a example that will differentiate between our algorithm and Kung's algorithm. Let we have some solutions in which only one solution dominate all other solutions. Since this solution is better than all solutions when we apply sorting it will come at the starting of the list. When we apply both the algorithms Kung's and Proposed algorithm on the sorted list of this problem, then for this case time complexity of our algorithm will be $\mathrm{O}$ (nlongn) where as The complexity of Kung's Algorithm will be $\mathrm{O}\left(\mathrm{n}^{2}\right)$.So our algorithm will always work faster than Kung's algorithm.

\subsection{Detail of Algorithm:}

To make the algorithm clear, consider the example taken from Jun Du's paper, we illustrate the working of above steps on this example. First, let's take out an MOO example with 10 solutions and 4 objectives. The following Table 1 presents the 4 objective function values (O1-O4) for 10 solutions (P1-P10).

Table 1: Objective Function Values

\begin{tabular}{|l|l|l|l|l|l|l|l|l|l|l|}
\hline $\begin{array}{l}\text { Obj. } \\
\text { Func. }\end{array}$ & P1 & P2 & P3 & P4 & P5 & P6 & P7 & P8 & P9 & P10 \\
\hline O1 & 0.94 & 0.35 & 0.76 & 0.88 & 0.39 & 0.86 & 0.27 & 0.91 & 0.73 & 0.53 \\
\hline O2 & 2934 & 3599 & 2780 & 1998 & 3476 & 3331 & 2597 & 2318 & 3273 & 4055 \\
\hline O3 & 5.3 & 6.6 & 5.4 & 8.0 & 8.7 & 7.9 & 9.1 & 2.1 & 4.9 & 7.7 \\
\hline O4 & 289 & 45 & 23 & 598 & 444 & 99 & 188 & 239 & 177 & 328 \\
\hline
\end{tabular}

According to the step 1 of the algorithm, we first sort solutions in descending order of their first objective function value therefore sorted list will contain $\{\mathrm{P} 1, \mathrm{P} 8, \mathrm{P} 4$, P6, P3, P9, P10, P5, P2, P7 \}. We take first solution of this list and add this solution to set S1.Now, we initialize a set S1 and put the first element P1 of the list to the set S1. Next we compare second solution P8 from set S1, as P1 dominate P8 so discard this solution and go for third solution P4. As the set $\mathrm{S} 1$ contains only one solution P1, so we need only one comparison to decide the category of P4. As $\mathrm{P} 1$ is non dominated to $\mathrm{P} 4$, so we update the initial set and now set $\mathrm{S} 1$ contains two solutions (P1, P4). We have to make two comparisons for P6, this solution is non dominated to both the solutions so we update the set $\mathrm{S} 1$ to (P1, P4, P6). For P3, we check its dominance with the set (P1, P4, P6), it is dominated by P6, so we discard this point. Similarly for other solutions we can repeat the same process

\section{Table1: Running time analysis of Jun du and proposed algorithm for Four Objective Functions}

\begin{tabular}{|c|c|c|c|c|}
\hline \multirow{2}{*}{$\begin{array}{l}\text { M (No. of } \\
\text { Objective } \\
\text { Functions) }\end{array}$} & \multirow{2}{*}{$\begin{array}{c}\text { N (Population } \\
\text { Size) }\end{array}$} & \multicolumn{2}{|c|}{ Running Time } & \multirow{2}{*}{$\begin{array}{l}\text { Q (No. of Non } \\
\text { dominated } \\
\text { Solutions) }\end{array}$} \\
\hline & & $\begin{array}{l}\text { Jun du's } \\
\text { Algorithm }\end{array}$ & $\begin{array}{l}\text { Proposed } \\
\text { Algorithm }\end{array}$ & \\
\hline \multirow{4}{*}{4} & \multirow{3}{*}{5000} & 885.4940 & 204.3920 & 737 \\
\hline & & 871.2670 & 183.0970 & 675 \\
\hline & & 443.1880 & 97.6710 & 737 \\
\hline & & 1463.5 & 317.5350 & 956 \\
\hline
\end{tabular}

\section{EXPERIMENTAL RESULTS AND COMPLEXITY ANALYSIS:}

To find a non-dominated set of a multi objective optimization problem, Comparison of Jun du's algorithm and proposed algorithm are performed on a computer with Intel core ${ }^{\mathbf{T M}} 2$ 1.60G Hz CPU and $1 \mathrm{~GB}$ memory. Running time of the algorithms is taken as the criterion to evaluate the efficiency. Various objectives number and solutions number are set for test.

The objective function values are generated randomly. To remove experimental error, comparisons are performed more than once. Tables 1 and 2 contain the experiments results, where $\mathrm{M}$ is objective number, $\mathrm{N}$ is population size and Q is the size of non dominated set. From the table, it is clear that our algorithm is more efficient than Jun du,s algorithm. 


\begin{tabular}{|c|c|c|c|c|}
\hline \multirow[t]{2}{*}{4} & \multirow[t]{2}{*}{10000} & 1313.2 & 305.8380 & 916 \\
\hline & & 1353.1 & 308.8800 & $\overline{918}$ \\
\hline \multirow{3}{*}{4} & \multirow{3}{*}{20000} & 3543.3 & 1010.5 & 1304 \\
\hline & & 3674.7 & 1017.7 & 1317 \\
\hline & & 3724.5 & 1026.2 & 1354 \\
\hline 4 & 50000 & 9029.1 & 5583.5 & 1872 \\
\hline
\end{tabular}

Table 2: Running time analysis of Jun du and proposed algorithm for Seven Objective Functions

\begin{tabular}{|c|r|r|r|r|}
\hline \multirow{2}{*}{\begin{tabular}{c} 
M (No. of $\begin{array}{c}\text { Objective } \\
\text { Functions) }\end{array}$ \\
\cline { 3 - 4 }
\end{tabular}} & $\begin{array}{c}\text { N (Population } \\
\text { Size) }\end{array}$ & \multicolumn{2}{|c|}{ Running Time } & \multirow{2}{*}{$\begin{array}{c}\text { (No. of Non } \\
\text { dominated } \\
\text { Solutions) }\end{array}$} \\
\cline { 3 - 5 } & \multirow{2}{*}{5000} & $\begin{array}{c}\text { Jun du's } \\
\text { Algorithm }\end{array}$ & $\begin{array}{c}\text { Proposed } \\
\text { Algorithm }\end{array}$ & 4263 \\
\cline { 3 - 5 } & & 1035.5 & 223.5940 & 675 \\
\cline { 3 - 5 } & \multirow{2}{*}{10000} & 1011.4 & 209.0090 & 697 \\
\cline { 3 - 5 } & & 1027.6 & 209.9300 & 918 \\
\cline { 3 - 5 } & & 3427.7 & 1001.6 & 917 \\
\hline \multirow{2}{*}{7} & 3546.3 & 1010.7 & 926 \\
\hline
\end{tabular}

Table3: Running time analysis of Jun du and proposed algorithm for Ten Objective Functions

\begin{tabular}{|c|r|r|r|r|}
\hline \multirow{2}{*}{$\begin{array}{c}\text { M (No. of } \\
\text { Objective } \\
\text { Functions) }\end{array}$} & \multirow{2}{*}{$\begin{array}{c}\text { (Population } \\
\text { Size) }\end{array}$} & \multicolumn{2}{|c|}{ Running Time } & \multirow{2}{*}{$\begin{array}{c}\text { (No. of Non } \\
\text { dominated } \\
\text { Solutions) }\end{array}$} \\
\cline { 3 - 4 } & & $\begin{array}{c}\text { Jun du's } \\
\text { Algorithm }\end{array}$ & $\begin{array}{c}\text { Proposed } \\
\text { Algorithm }\end{array}$ & 118 \\
\hline \multirow{3}{*}{10} & \multirow{2}{*}{5000} & 813.9270 & 185.3600 & 1106 \\
\cline { 3 - 4 } & \multirow{2}{*}{10} & 792.1040 & 198.5720 & 1126 \\
\cline { 3 - 4 } & \multirow{2}{*}{10000} & 811.4530 & 187.4750 & 1567 \\
\cline { 3 - 4 } & & 9167.1 & 2667.9 & 2131 \\
\hline
\end{tabular}

\subsection{Complexity analysis}

(i)

The best case complexity of the proposed algorithm will be $O(N \operatorname{LogN})$ this can be calculated as follows.

In step 1 the time taken by quick sort will be NLogN per List. For the best case the number of solutions in set $\mathrm{S} 1$ will be one, so in total $\mathrm{N}$ (one for each solution) comparisons will be required to find the non dominated set. This condition occurs when all non dominated fronts contain only one solution.

(ii) The worst case complexity of the proposed algorithm will be $O\left(M(N)^{2}\right)$ this can be calculated as follows.

In step 1 the time taken by quick sort will be NLogN per List. For this case there will be no dominated solution in the list. So to check non dominance of a solution, all solutions of $\mathrm{S} 1$ is to be checked.

\section{CONCLUSION:}

The algorithm proposed in this paper could find nondominated set efficiently by two steps: sorting step and deleting step. The time complexity analysis shows that this algorithm is better than any other algorithm in its best case analysis. Also in average case its complexity is same as of Kung's algorithm which has better complexity in comparison of other traditional algorithm. The idea can be extended to provide non dominated sorting of the population. 


\section{REFERENCES:}

[1]. Jun Du, Zhihua Cai and Yunliang Chen," A Sorting Based Algorithm for Finding NonDominated Set in Multi-Objective Optimization" Third International Conference on Natural Computation (ICNC 2007)

[2] K. Deb, "Multi-objective Optimization Using Evolutionary Algorithms," JOHN WILEY\&SONS, LTD, 2001 pp.33-43, 2000.

[3]. K. Deb, S. Agrawal, A. Pratap, and T. Meyarivan. Afast elitist non-dominated sorting genetic algorithm for multi-objectv e optimization: NSGA-II. In Proceeding of the $6^{\text {th }}$ International Conference on Parallel Problem Solving from Nature, Pages 849-858, 2000.

[4]. L. Ding. S.Zeng. and L. Kang, "A fast algorithm on finding the non-dominated set in multiobjectve optimization.", In Proceedings of International Conference on Evolutionary Computation, pages 2565-2571, 2003

[5] A. Freitas," A critical review of multi-objective optimization in data mining: a position paper. “,SIGKDD Explorations, 6(2): 77-86, 2004.

[6] J. Knowles and D. Corne,"Reducing local optima in single-objective problems by multiobjectivization", In Proceedings of the $1^{\text {st }}$ International Conference on Evolutionary MultiCriterion Optimization, pages 269-283, 2001.

[7] H. Kung. F. Luccio, and F. Preparata,"On finding the maxima of a set of vectors.", Journal of the Association Computing Machinery, 22(4) : 469476, 1975.

[8] E. Zitzler, M. Laumanns, and L. Thiele,"Spea2: Improving the strength Pareto evolutionary algorithm for multiobjective optimization.", In Proceedings of the Evolutionary Methods for Design, Optimization, and Control, 19-26, Barcelona, Spain, 2002.

[9]. C. Fonseca, M. and P. J. Fleming "Genetic algorithms for multiobjective optimization: Formulation, discussion and generalization," In S. Forrest (Ed.), Proceedings of the Fifth International Conference on Genetic Algorithms, San Mateo, California, pp. 416C423. Morgan Kaufmann. , 2003

[10]. J. Horn, and N. Nafpliotis "Multiobjective optimization using the niched Pareto genetic algorithm," IlliGAL Report 93005, Illinois Genetic Algorithms Laboratory, University of Illinois, Urbana, Champaign.,1993
[11]. J. Horn, N. Nafpliotis, and D. E. Goldberg" A niched Pareto genetic algorithm for multiobjective optimization," In Proceedings of the First IEEE Conference on Evolutionary Computation, IEEE World Congress on Computational Computation, Volume 1, Piscataway, NJ, pp. 82C87. IEEE. , 1994

[12]. R. C. Purshouse, P. J. Fleming " The Multiobjective Genetic Algorithm Applied to Benchmark Problems-an Analysis," Research Report No. 796. Department of Automatic Control and Systems Engineering University of Sheffield, Sheffield, S1 3JD, UK. 2001

[13]. J. D. Schaffer "Multiple objective optimization with vector evaluated genetic algorithms," In J. J. Grefenstette (Ed.),Proceedings of an International Conference on Genetic Algorithms and Their Applications, Pittsburgh, PA, pp. 93C100. sponsored by Texas Instruments and U.S. Navy Center for Applied Research in Artificial Intelligence (NCARAI), 1985

[14]. N. Srinivas and K. Deb , " Multiobjective optimization using non-dominated sorting in genetic algorithms," Evolutionary Computation 2(3), 221C248, 1985

[15]. A. Tiwari and R. Roy(2002) "Generalised Regression GA for Handling Inseparable Function Interaction: Algorithm and Applications," Proceedings of the seventh international conference on parallel problem solving from nature. ( PPSN VII). Granada, Spain

[16]. E. Zitzler" Evolutionary Algorithms for Multiobjective Optimization: Methods and Applications," Ph. D. thesis, Swiss Federal Institute of Technology (ETH) Zurich, Switzerland. TIK-Schriftenreihe Nr. 30, Diss ETH No. 13398, Shaker Verlag, Aachen, Germany, 1999

[17]. E. Zitzler, M. Laumanns and L. Thiele," SPEA2: Improving the Strength Pareto Evolutionary Algorithm," TIK-Report 103. ETH Zentrum, Gloriastrasse 35, CH-8092 Zurich, Switzerland. 1999. 